\title{
Artificial intelligence for IoT systems
}

\author{
Andrés Muñoz ${ }^{\mathrm{a}}$, Juan Carlos Augusto ${ }^{\mathrm{b}}$, Vincent Tam ${ }^{\mathrm{c}}$ and Hamid Aghajan ${ }^{\mathrm{d}}$ \\ a Polytechnic School, Universidad Católica de Murcia, Spain \\ ${ }^{\mathrm{b}}$ Department of Computer Science and Research Group on Development of Intelligent Environments, Middlesex \\ University, $U K$ \\ ${ }^{\mathrm{c}}$ Department of Electrical and Electronic Engineering, Faculty of Engineering, The University of Hong Kong, \\ China \\ ${ }^{\mathrm{d}}$ imec, IPI, Department of Telecommunications and Information Processing, Gent University, Belgium
}

\section{This thematic issue}

We begin the year 2020 with this issue focused on the topic of "Artificial Intelligence for IoT Systems". Undoubtedly, the Internet of Things paradigm has brought about a significant revolution in the past decade and has served to foster the development of intelligent environments [2]. Whereas the hardware components (i.e. sensors and actuators) and communication protocols of IoT systems have been extensively studied in the last few years, this new decade brings about a series of challenges in the application and development of intelligent techniques and algorithms for the processing of the data collected by these systems [1]. To achieve a truly connected and intelligent world, capable of adapting to the needs of users, we not only need high performance systems but also systems that are genuinely adaptive and that understand what is happening in their environment and act consistently and in line with the users' desires.

We are pleased to introduce this Thematic Issue as an opportunity to highlight the importance of this topic and contribute to its progress. We wish to thank our colleagues Dr. Imran Sarwar Bajwa (University Bahawalpur), Dr. George Roussos (University of London), Dr. Anna Helena Reali Costa (University of São Paulo) and Dr. William Grosky (University of Michigan) for organizing the content of this issue and bringing their research experience in the area of artificial intelligence for IoT systems to achieve this Thematic Issue for our Journal.

\section{Upcoming issues}

The following is the list of upcoming issues of JAISE:

- March 2020: Regular Issue.

- May 2020: Thematic Issue on "Selected Papers from Intelligent Environments 2019".

- July 2020: Regular Issue.

- September 2020: Regular Issue.

More information on the call for papers for future thematic issues is available on the webpage of JAISE at: http://www.iospress.nl/journal/journalof-ambientintelligence-and-smart-environments/

\section{References}

[1] M. Gams, I.Y. Gu, A. Härmä, A. Muñoz and V. Tam, Artificial intelligence and ambient intelligence, Journal of Ambient Intelligence and Smart Environments 11(1) (2019), 71-86. doi:10. 3233/AIS-180508.

[2] C. Gomez, S. Chessa, A. Fleury, G. Roussos and D. Preuveneers, Internet of things for enabling smart environments: A technology-centric perspective, Journal of Ambient Intelligence and Smart Environments 11(1) (2019), 23-43. doi:10. 3233/AIS- 180509. 